\title{
Hypersonic Aerothermodynamics: Past, Present and Future
}

\section{Chul Park*}

Department of Aerospace Engineering, Korea Advanced Institute of Science and Technology, 373-1 Guseong-dong, Yuseong-gu, Daejeon 305-701, Korea

\begin{abstract}
This is a written version of the keynote speech delivered at the International Symposium on Hypersonic Aerothermodynamics - Recent Advances held in Bangalore, India, from December $6^{\text {th }}$ to $10^{\text {th }}, 2012$. In this document, what was accomplished in the past, the present status, and what is expected in the future in the field of hypersonic aerothermodynamics are reviewed. Solved problems are categorized into four items; unsolved problems into twelve items, and emerging problems into four items. Removing one degree uncertainty in trim angle of attack, studying the thermochemical phenomena in a hydrogen-heliummethane mixture, and entry flights of meteoroids are cited as the tasks for the future.
\end{abstract}

Key words: Hypersonics, aerothermoynamics, chemical kinetics, two-temperature model

\section{Introduction}

From December $6^{\text {th }}$ to $10^{\text {th }}$ in 2012 , in Bangalore, India, International Symposium on Hypersonic Aerothermodynamics - Recent Advances was held. The acronym of the symposium is ISHARA, which is the name of an Indian goddess who points the direction. It is an apt name of this symposium because the purpose of the symposium is to point the future direction of this branch of technology.
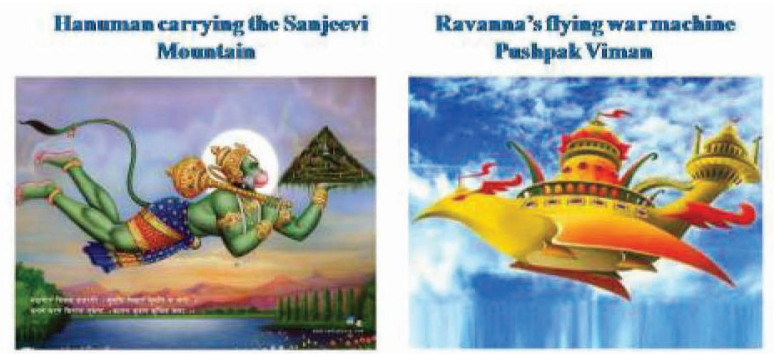

Fig. 1. Hanuman bringing the Samjeeve mountain home and Ravanna's flying machine Pushpak Viman (Ramayana, circa 1300 BC).

The theme of this symposium, high speed flight, is also well engrained in the host country's tradition. According to Ramayana, one of the two sacred writings of Indian philosophy, there was a gracious queen named Sita long time ago. She became ill, and needed a medicine, an herb growing in a certain mountain. The faithful friend Hanuman goes to the mountain, but does not know which is the right herb. So he brings the whole of this mountain home. He hurries and flies at a hypersonic speed, as shown in the left side figure of Figure 1. But there is a bad person also: the evil king Ravanna, who lives in a remote island, builds a secret flying machine to kidnap the gracious queen, as shown in the right of Figure 1. In this way, high speed flight was deeply associated with the way of thinking of humans.

\section{Past Accomplishments}

In modern times, in the second half of the twentieth century, humans began a concerted effort to reach to the sky, starting with the Apollo project. Hypersonic aerothermodynamics was the key to that human endeavor: the project produced the very aerothermodynamics itself.

On return from the moon, the Apollo vehicle had to enter the atmosphere with astronauts in it. The vehicle, Apollo Command Module, was made into a blunt capsule shape hitherto unknown in the community of flying. The Module

This is an Open Access article distributed under the terms of the Creative Commons Attribution Non-Commercial License (http://creativecommons.org/licenses/by$\mathrm{nc} / 3.0 /$ which permits unrestricted non-commercial use, distribution, and reproduction in any medium, provided the original work is properly cited. 
was geometrically axisymmetric, but the center of gravity was placed at a point away from the axis of symmetry. Because of this center-of- gravity offset, the Module flew at a finite angle of attack during its entry flight. As a result, a lift force was produced. The lift force was directed downward in the beginning, and was flipped over to point upward later. By doing so, the Module could fly a prolonged flight trajectory that maintained the vehicle's deceleration to a level bearable by the astronauts. If this was not done, the Module faced the danger of either skipping out into the space never to return or decelerate so hard that the astronauts inside the Module would be crushed to death.

In order to adhere to this prescribed flight trajectory, it was necessary for the Module to have a certain lift coefficient. To do so, the Module had to aerodynamically trim at a certain prescribed angle of attack. Before an Apollo vehicle went to the moon, both wind tunnel and flight experiments were made to determine this trim angle of attack. There was a difference in the trim angle of attack obtained by these two methods. Up to $4^{\circ}$ of difference was found between the two as shown in Figure 2 [1]. The center of gravity was adjusted by considering this flight data. Even so, in the first return from the moon, the Command Module landed some $200 \mathrm{~km}$ away from the predicted point in the Pacific Ocean because of this trim angle of attack uncertainty.

This shift in trim angle of attack has later been determined to be due to the change in the shape of the bow shock wave over the entry vehicle, which was in turn due to the high temperature real gas effect, i.e. vibrational excitation, dissociation, and ionization. The science of this high temperature real gas effect is now called aerothermodynamics. Today, one can numerically reproduce this phenomenon using computational fluid dynamics (CFD) to within about $1^{\circ}$ in trim angle.

Later, when the Space Shuttle started to fly, the same phenomenon happened during the entry flight. The vehicle was supposed to fly at a trim angle of attack of $40^{\circ}$ during its

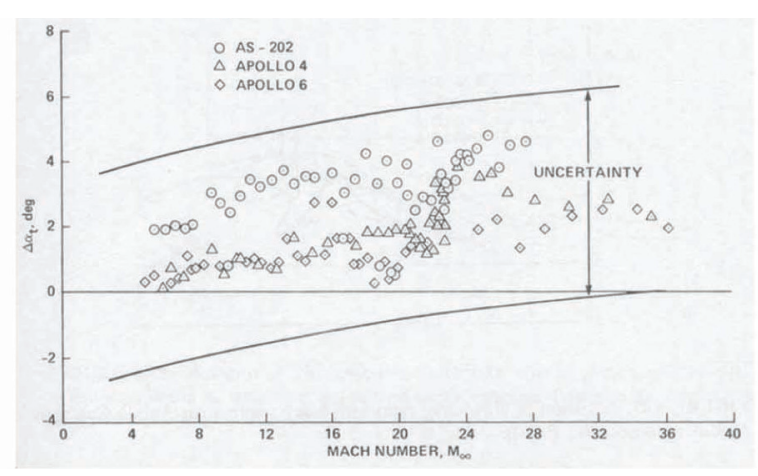

Fig. 2. Departure of the flight trim angle of attack from the wind tunnel-determined trim angle of attack for Apollo [1] hypersonic portion of the entry flight. But the vehicle started to pitch up. This nose-up pitching motion continued even when the body flap was deflected downward to its maximum extent. The vehicle's angle of attack reached 44o [2]. If it went further, the vehicle would have flipped over and been destroyed because the unprotected top side would have faced the hot high pressure shock layer flow. The location of the center of pressure deduced from the flight trim angle of attack is compared with the predicted value in Figure 3.

As was for Apollo vehicle, the shift in trim angle of attack was due to the high temperature real gas effect. Again, this phenomenon can be numerically recreated today with the use of CFD to within about $1^{\circ}$. Explaining these high temperature real gas effects on aerodynamic characteristics was the first major accomplishment of aerothermodynamics.

Next important accomplishment of aerothermo-dynamics concerns the so-called nonequilibrium radiation overshoot phenomenon. For the Apollo vehicle, the heat transfer to the forebody was due partly to the radiation emitted by the hot shock layer. To determine the intensity of this radiative heat transfer, shock tube experiments were conducted. A normal shock wave was produced in a shock tube to a speed of up to $11 \mathrm{~km} / \mathrm{s}$ occurring during the Apollo's entry flight, and the

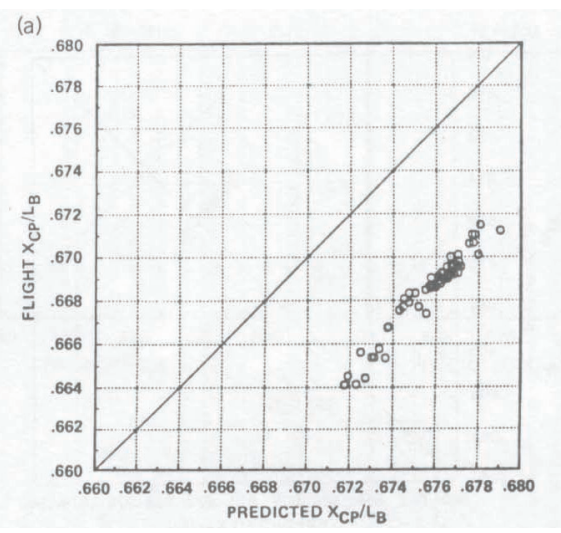

Fig. 3. The flight center of pressure compared with the predicted for the Space Shuttle [2]

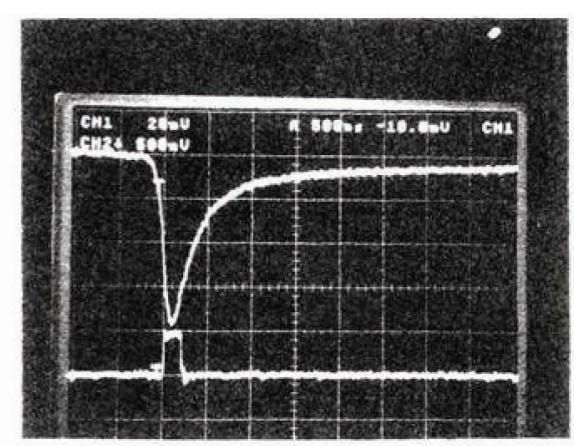

Fig. 4. Radiation intensity behind a normal shock wave [3] 
intensity of the radiation emitted by the hot gas behind the shock wave was measured. Surprisingly, the radiation was strongest at a short distance behind the shock wave as shown in Figure 4 [3]. The peak intensity was much larger than that in equilibrium. The distance, or equivalently the time, to this nonequilibrium peak and the time to reach the equilibrium value were determined and plotted as shown in Figure 5.

In order to explain this phenomenon, the physics of radiation had to be studied and combined with fluid mechanics. This study led to the so-called two-temperature nonequilibrium model. By using this model, the observed characteristic times were numerically recreated successfully

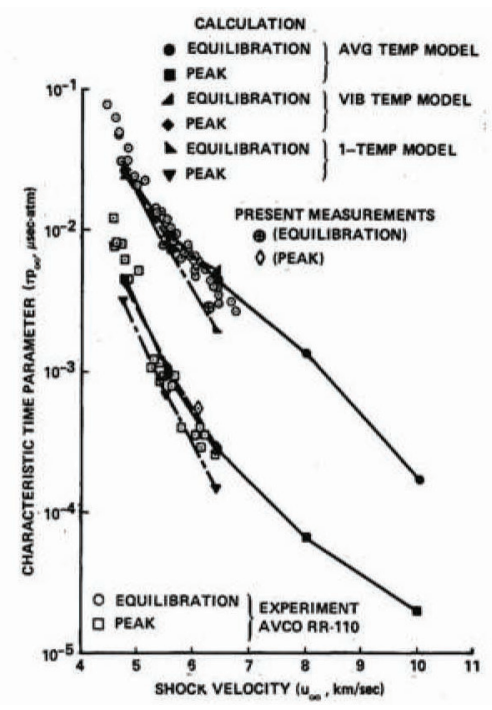

Fig. 5. The time to the peak and time to equilibration of the radiation behind a normal shock wave [4]
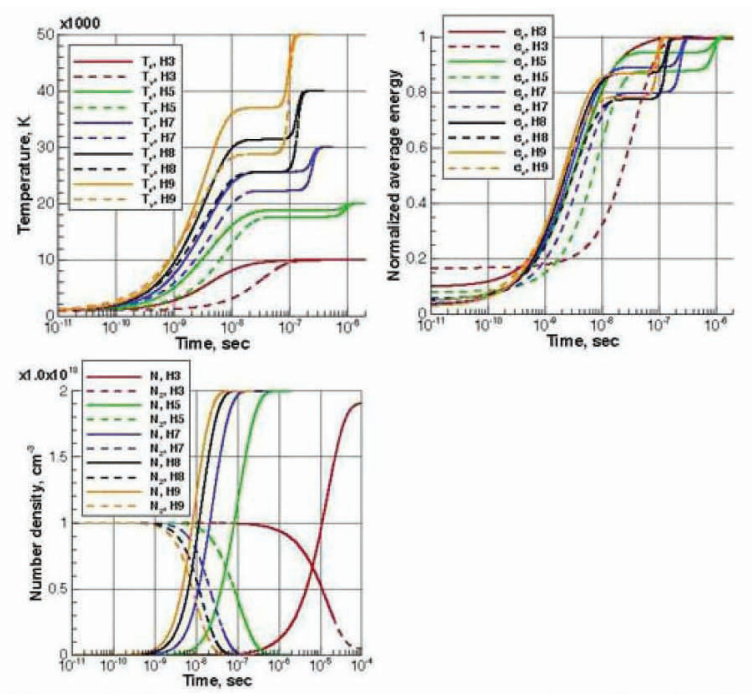

Fig. 6. Temperature, average energy, and number density behind a normal shock wave in nitrogen [5] as shown in Figure 5 [4]. The two-temperature model stipulated that the rotational mode is in equilibrium with the translational mode and the vibrational mode is in equilibrium with the electronic mode and the translational mode of the electrons.

Next important accomplishment is in the field of molecular dynamics. It is possible today to calculate in detail the motion of individual atoms in a molecular collision by integrating conservation equations. From this, one can determine the rate of transition from one internal (vibrational, rotational, or electronic) state to another internal state, i.e. state-tostate transition rate. Then the number density of atoms and molecules in each energy state can be calculated by integrating a system of differential equations known as Master equation. This enables determination of vibrational and rotational energies of each molecules. In Figure 6, one such example, taken from Kim et al [5] is shown.

One important observation can be made in Figure 6: there is a period from about $10^{-4}$ to $10^{-2}$ seconds during which temperatures do not vary but number densities vary. This is the so-called quasi-steady-state (QSS) period in which the rate of transitions into an internal state is balanced by the rate of transitions out of the state. Most chemical reactions occur during this QSS period, as can be seen by the change in number densities. The experimentally-determined reaction rates are those observed during this period.

\section{Unsolved Problems}

Still there are many more unsolved problems than solved problems. The first of these unsolved problems is not as much an unsolved problem but cavalier attitude of the scientists. It concerns the definition of reaction rates. Many researchers think that the rate of reaction is a sum of all stateto-state transitions from an initial state to a final state. It is not. A reaction rate is a sum of only those components of the state-to-state transition rates proportional to the initial state density under a quasi-steady-state condition. The former is called one-way rate and the latter is called QSS rate. The oneway-rates do not satisfy law of mass action; the QSS rates do. It is the quasi-steady-state rates that are determined experimentally. The QSS rates are about one-third of the one-way rates in dissociation. In ionization, the ratio is of the order of one-ten thousandth. The problem is described succinctly by Park [6]. But, seemingly majority of aerospace engineers cavalierly skips over this portion of the book.

The second unsolved problem concerns the so-called double-done flow. The flow around a double cone, shown in Figure 7 , has been studied both experimentally and 
theoretically [7]. This flow-field involves boundary layer phenomenon, separation, shock-shock interaction, and shock-boundary layer interaction. When there is no chemical reaction, the calculated values of pressure and heat transfer rate distributions agree with the experimental data closely. However, when chemical reactions occur, agreement is not so good. This discrepancy has not yet been explained.

The third unsolved problem is on the shock stand-off distance over a sphere at intermediate hypersonic speeds, i.e., speed range of 2500 to $4000 \mathrm{~m} / \mathrm{s}$, in air. Shock stand-off distance was measured in a ballistic range where there was no question as to the chemical state of the free-stream. The measured shock stand-off distance was slightly larger than the calculated distance, as shown in Figure 8 [8]. This occurs if the gas under study contains significantly concentration of molecules, that is, if the flight speed is not very high. If shock stand-off distance is not correctly calculated, shock shape and consequently pressure is not correctly calculated. The phenomenon may be due to rotational nonequilibrium (Furudate et al, 1999).

The fourth unsolved problem also concerns shock standoff distance, but not as much the process behind the shock wave but the nozzle flow. Shock stand-off distance of a sphere cone was measured in a shock tunnel, in the test section located at the end of a diverging nozzle, in carbon

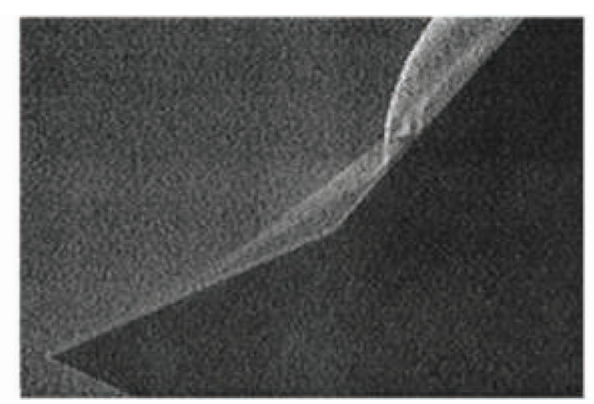

Fig. 7. Flow-field over a double-cone [7]

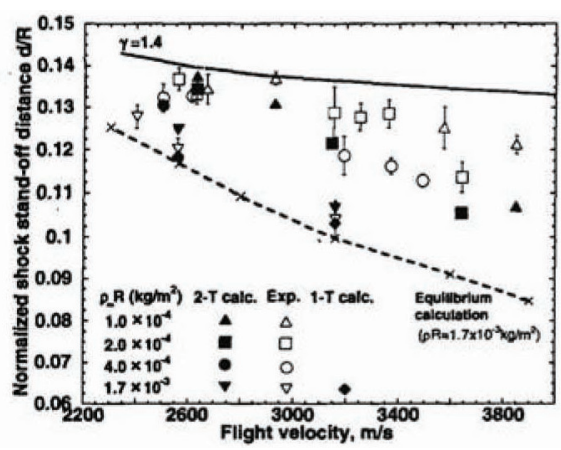

Fig. 8. Comparison between the measured and calculated shock stand-off distances for sphere in the intermediate hypersonic speed range [8] dioxide. The measured shock stand-off distance was much larger than the calculated [7], as shown in Figure 9.

But, when the experiment was repeated in an expansion tube, in which gas does not go through expansion from a high pressure, high temperature state, the shock stand-off distance was much smaller and agreed with a calculation, as shown in Figure 10 [7]. Therefore, the large shock standoff distance in Figure 9 is due to chemical freezing in the nozzle. The CFD calculation shown in Figure 9 accounted for the vibrational freezing in the nozzle. The only way this discrepancy could occur is if rotational mode is also frozen in the nozzle. But such rotational freezing is not yet known.

The fifth unsolved problem concerns the rotational temperature behind a normal shock wave. Sharma et al [3] measured the vibrational and rotational temperatures behind a normal shock wave in a shock tube spectroscopically. The results were compared with a calculation made using the two-temperature model. Vibrational temperature was found to agree roughly with the calculation. But the rotational temperature, which, according to the two-temperature model, should be the same as the translational temperature and hence high, was found to be low, as shown in Figure 11. The same was true in the experiment by Fujita et al [9], shown in Figure 12.

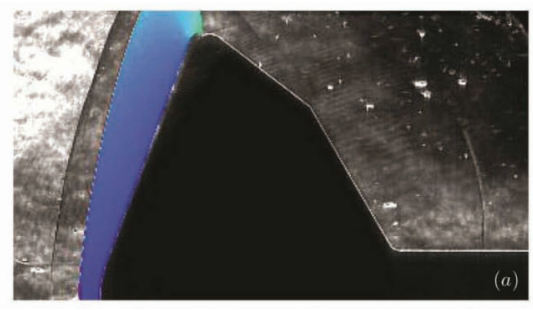

Fig. 9. Comparison between the measured and calculated shock shapes, measurement made in a shock tunnel, in carbon dioxide [7]
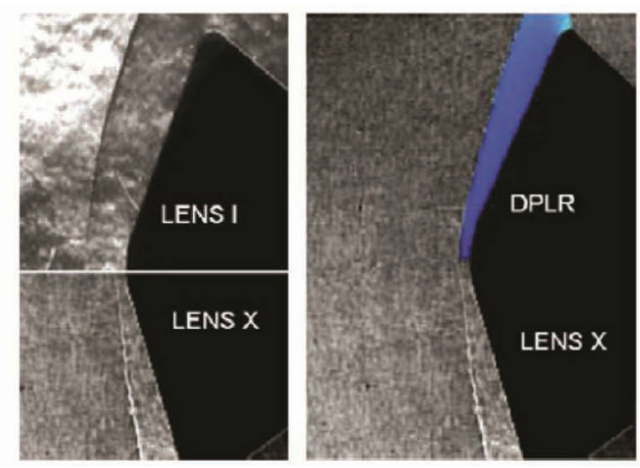

Fig. 10. Comparison between the shock shape taken in a shock tunnel (LENS1) and that taken in an expansion tube (LENS X), and computer calculation (DPLR) [7] 
The sixth unsolved problem is about the distribution of rotational states in an expanding flow. The measured distribution was not at all Boltzmann: the lower states were found to be populated following a very low Boltzmann temperature while high states were found to be populated following a substantially higher Boltzmann temperature, as shown in Figure 13 [10]. The two-temperature model assumes that the rotational mode is in equilibrium with the translational mode. Figure 13 shows that the assumption is not correct.

The seventh unsolved problem also concerns nonequilibrium of the rotational mode in a molecule. For the rotational states up to about 15 , the rotational transition rates have been measured for $\mathrm{N}_{2}$. When these measured transition rates are extrapolated to higher states, one obtains the times needed for rotational equilibration, i.e.

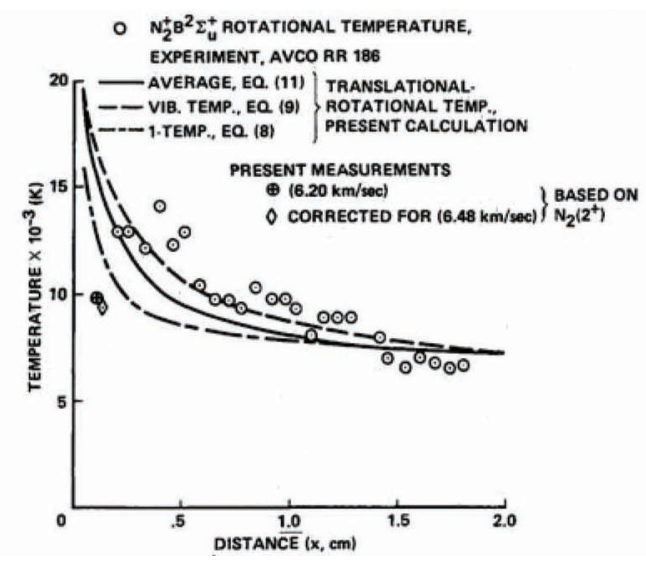

Fig. 11. Comparison between the measured and calculated vibrational and rotational temperatures behind a shock wave in the experiment by Sharma et al [3]

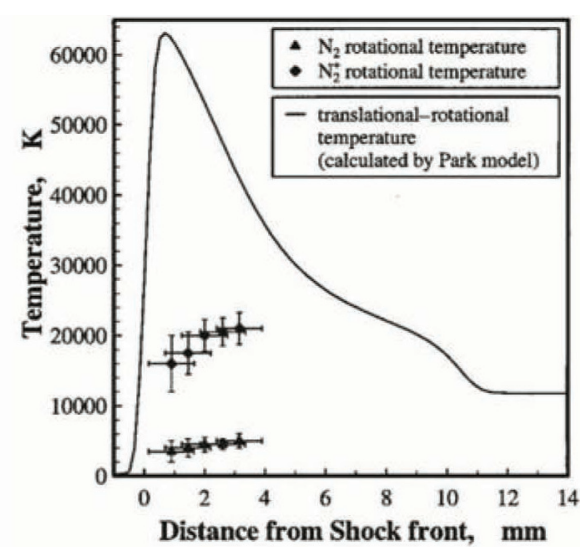

Fig. 12. The measured vibrational temperature and rotational temperature behind a normal shock wave compared with the calculated translational temperature in the experiment by Fujita et al [9] rotational equilibration times, that becomes very small at high temperatures, as shown in Figure 14 [11]. Very recently, Kim et al [5] have shown, through a molecular dynamics calculation, that indeed this is true, as shown in Figue 15.

The eighth unsolved problem is about the ablation of Galileo Probe vehicle during its entry flight into the planet Jupiter. The vehicle was thermally protected with a thick heatshield made of carbon-phenolic. During the entry flight, the extent of the recession of this heat shield was measured. The measured recession was considerably smaller than predicted at the stagnation point, and was considerably larger at the frustum, as shown in Figure 16 [12].

The overestimation of surface recession at the stagnation point was explained by Park [13], as being due to the inaccuracy in calculating the equilibrium composition. For the frustum region, Matsuyama et al [12], presented a theory based on complicated interaction of turbulence and radiative transfer. Matsuyama's thesis needs further verification

The ninth unsolved problem concerns the nonequilibrium behavior in a $\mathrm{H}_{2}+\mathrm{He}+\mathrm{CH}_{4}$ mixture [14]. The outer planets,

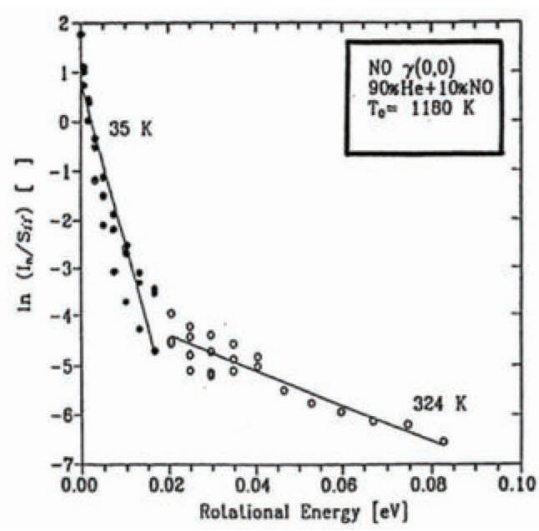

Fig. 13. Distribution of rotational states in an expanding flow (Dankert, 1998)

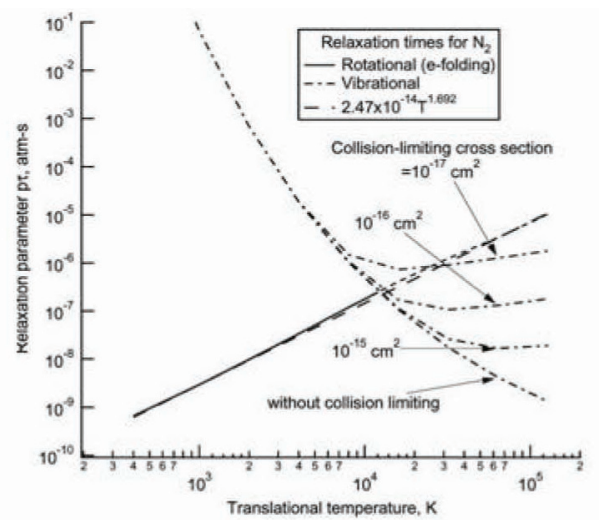

Fig. 14. The rotational collision times at high temperatures for nitrogen predicted by Park, 2004 
i.e., Jupiter, Saturn, Uranus, and Neptune, consist of this gas mixture. Leibowitz [15] and Livingston and Poon [16] measured the equilibration distance in $\mathrm{H}_{2}+\mathrm{He}$ mixtures. The measurement was made using an electric arc-driven shock tube, but the two groups used slightly different designs of the driver.

The relaxation distance determined by the two groups, Leibowitz [15] vs Livingston and Poon [16], differed by a factor of about three. Park [14] reconciled between the two sets by invoking the radiative heating of the test gas by the electrically-heated driver gas. Park's model is compared with Livingston-Poon data in Figure 17. Park [14] further theorized that the small amount of $\mathrm{CH}_{4}$ existing in the atmosphere of those outer planets significantly modifies the relaxation distance, as shown in Figure 17. Park's theory needs experimental verification.

The tenth unsolved problem concerns shock tube technology. Bogdanoff et al [17] made an effort to verify the accuracy of the measurement of Leibowitz and that by Livingston and Poon by conducting an experiment in an electric arc shock tube similar to that used by Leibowitz.

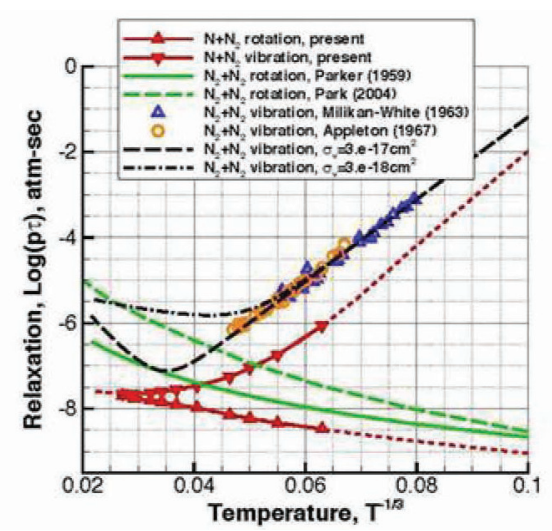

Fig. 15. Vibrational and rotational relaxation times calculated by Kim et al [5]

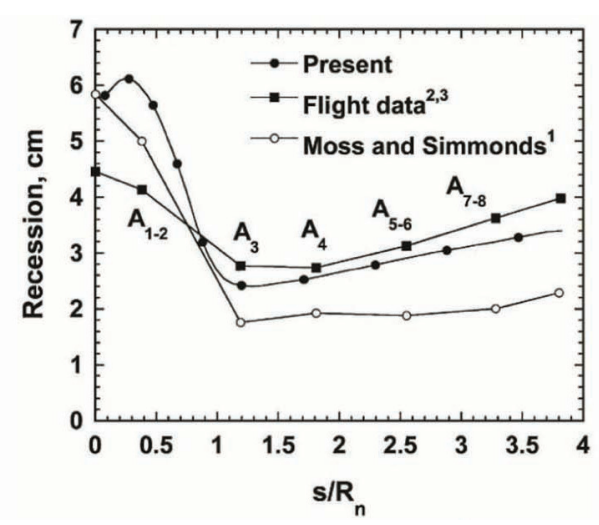

Fig. 16. Surface recessions of Galileo Probe (Matsuyama et al, 2005)
The result, shown in Figure 18, was surprising: the measured electron density was orders of magnitude larger than calculated. Through separate experiments, Bogdanoff et al confirmed that this ionization enhancement was due to the radiative heating of the test gas by the electrically-heated driver gas: the driver temperature was deduced to be about 40,000 K. Substantial electron density was found ahead of the shock wave, that is, precursor ionization was occurring.

Thus, the problem of simulating the entry flights into outer planets leads to a question as to whether one can produce the needed high shock speed, of about $25 \mathrm{~km} / \mathrm{s}$, in a shock tube. As we know it today, only electrical heating can heat the driver to produce the needed shock speeds.

This problem of ionization by radiation leads to the eleventh unsolved problem of not being able to predict the ionization behavior across a strong shock wave in general. Omura et al [18] measured electron density in a shock tube driven by an electric arc. To a great surprise, electron density was detected six meters ahead of the shock wave, as shown

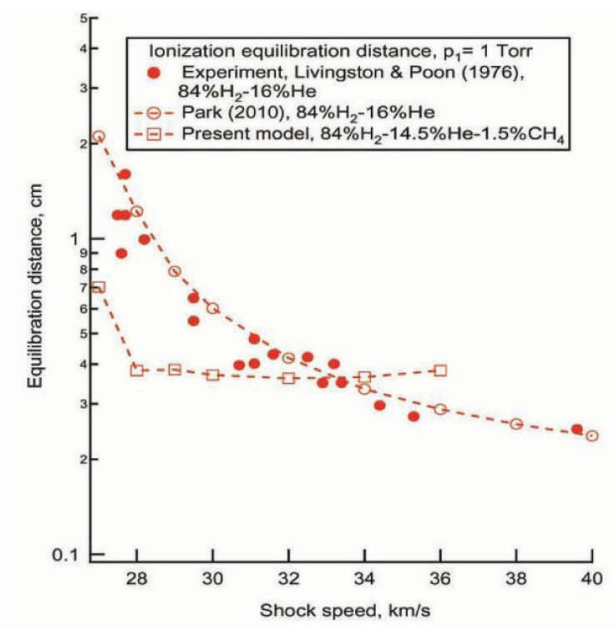

Fig. 17. Equilibration distance in a mixture of hydrogen, helium, and methane [14]

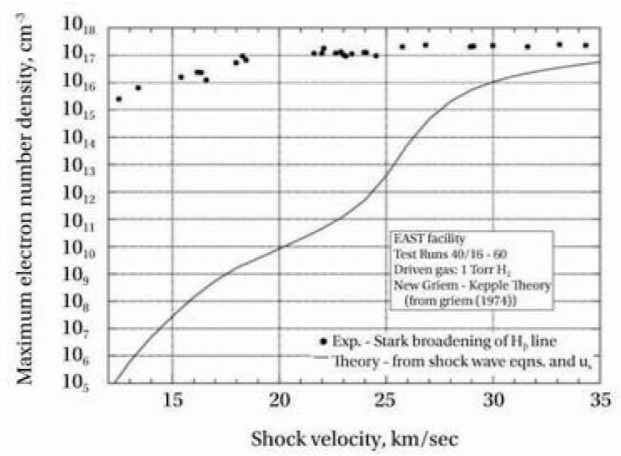

Fig. 18. The maximum electron density measured behind a shock wave in a $90 \% \mathrm{H}_{2}+10 \% \mathrm{He}$ mixture by Bogdanoff et al [17] measurement made in an electric arc shock tube 
in Figure 19.

Electron density was measured in the well-known RAM-C flight test [19]. A portion of the data obtained were analyzed and numerically recreated. But much of the flight data were left unexplained. In February of 1994, an experimental flight vehicle named OREX was flown by Japan. The vehicle, 3.4 meter in diameter, was made to reenter from a circular Earth orbit. Near the frustum of this vehicle was an electro-static probe. The electro-static probe data was analyzed by Wada et al [20].

The results are shown in Figure 20. As shown herein, the measured electron density values were about twice those calculated using the two-temperature model. Rather, the flight data agreed with a one-temperature model, which makes no sense. Thus, ionization phenomenon should be deemed at this time to be beyond our understanding.

The twelfth unsolved problem concerns ablation at high pressures. Through various experiments, it was established that a flow is laminar at the stagnation region of a blunt body no matter how rough the surface is. Ablation is a surface-

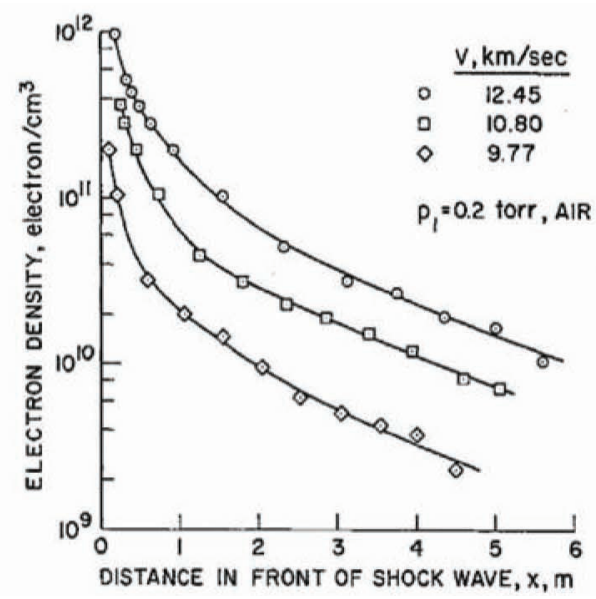

Fig. 19. Electron density ahead of the shock wave measured in an electric arc shock tube in air [18]

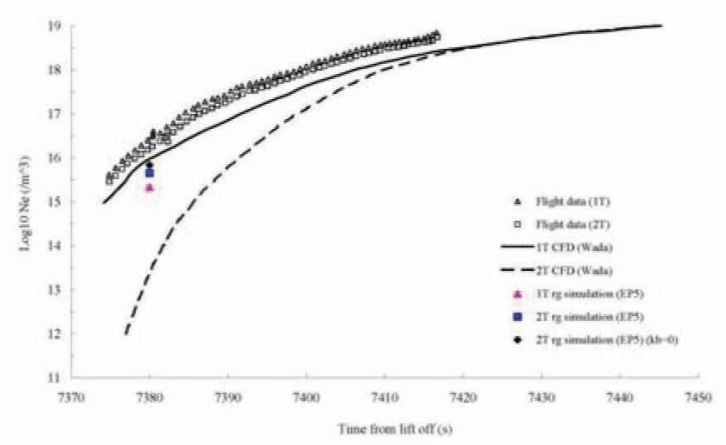

Fig. 20. Comparison between the measured and calculated electron densities in the OREX experiment [20] injection phenomenon. A laminar stagnation-point flow with injection can be calculated accurately. It is known well that heat transfer rate is reduced greatly by surface injection, in a phenomenon called convective blockage effect.

Rate of ablation can be determined by dividing the heat transfer rate by the effective heat of ablation. Heat of ablation is known to be typically about $20 \mathrm{MJ} / \mathrm{kg}$ for most carbonaceous heat-shield materials. When there is no ablation, heat transfer rate can be determined quite accurately using a formula such as by the Fay and Riddell. Because surface injection reduces heat transfer rate, when ablation occurs, the rate of ablation should be smaller than the value obtained by dividing the Fay-Riddell heat transfer rate by the heat of ablation.

But the experimental data are very different when stagnation pressure is high [21], as shown in Figure 21. In Figure 21, the curve marked LAMINAR is that obtained by dividing the Fay-Riddell heat transfer rate by the heat of ablation (assuming no ablation). Theoretically, surface recession rate should be smaller than this value. But the experimental data are higher. In fact, the discrepancy between the experimental data and the theoretical value ranges up to about 40 .

Park [21] reasoned that ablation phenomenon produces turbulence in the stagnation region. This injection-induced turbulence theory was used by Matsuyama [12] to explain the large surface recession in the frustum region of the Galileo Probe vehicle. This issue is worth pursuing further.

\section{Emerging Problems}

In addition to these unsolved problems, we aerothermodynamicists must pay attention to the newly

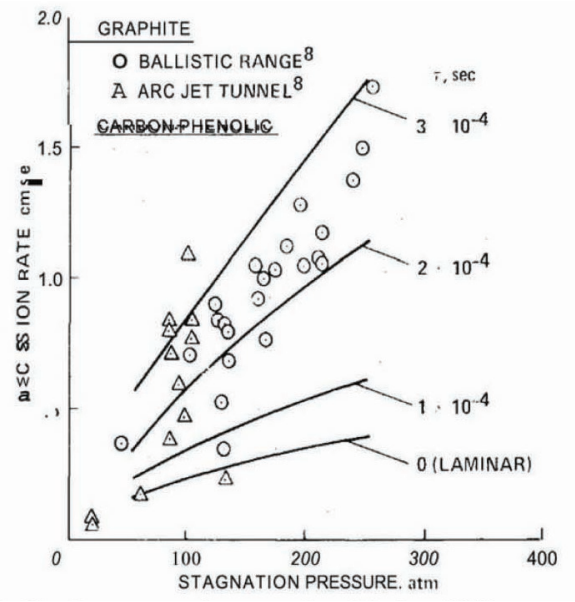

Fig. 21. Surface recession rate in ablation [21] 
emerging problems. The first of these emerging problems is with the space tourism. SpaceShip One and SpaceShip Two designed by the famous designer Burt Rutan for space tourism use a newly developed hybrid rocket engine. This hybrid rocket engine, which uses nitrous oxide as the oxidizer and paraffin as the fuel, has a fairly low specific impulse but is simple and safe. The vehicle takes off horizontally and lands horizontally, which is not as efficient as the conventional vertical takeoff method but safer. This safety and simplicity-minded design approach needs to be understood, appreciated, and emulated by the traditional hypersonic aerothermodynamicists.

The burden of this new approach is that the aerodynamic characteristics must be known accurately, because otherwise the passengers may be subject to excessive heat or deceleration. One degree of uncertainty in trim angle of attack is much too large for this case: the uncertainty must be reduced to a small fraction of one degree as for today's ocean-hopping subsonic jet liners.

The second important emerging problem concerns new thrust in planetary exploration focused on finding the origin of life. All living things on Earth are made up of amino acid molecules. There are two types of amino acid molecules, left-handed type, called L-alamine, and right-handed type, called D-alamine, differentiated by the location of nitrogen atom, shown in Figure 22. When amino acid molecules are synthesized in a ground-based laboratory, the two types are produced in equal proportions. Why then are we all made of one type, left-handed type, only?

Clue is found in space. Asteroids falling into Earth were found to contain also mostly the left-handed amino acid molecules. Right-handed types are preferentially destroyed by the infrared radiation of the sun. This molecule-killing infrared radiation is absorbed by Earth's atmosphere and therefore does not reach Earth's surface. This leads one to speculate that Earth's living things are made of the seeds

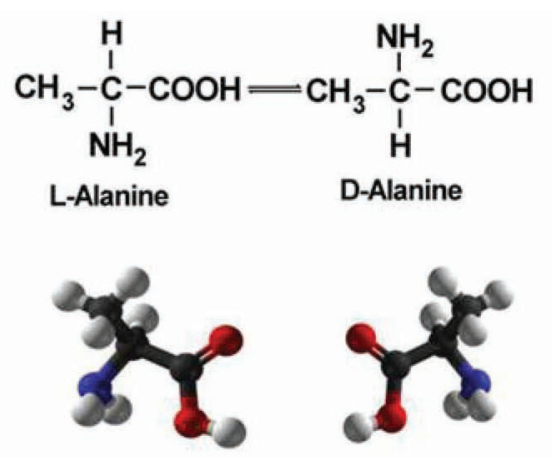

Fig. 22. Left-handed (L-alamine) and right-handed (D-alamine) amino acid molecules brought in by meteoroids. All meteoroids are heated in the entry process, and therefore, more delicate substances such as DNA molecules, if present, would be destroyed during the entry flight. Do the meteoroids in space contain DNA molecules as well as amino acid molecules?

To answer this fascinating question, one could do two things. First, we could fly to the outer planets and their moons. If these seeds of life came from out of the solar system, the outer planets would have more of them. They could be in the atmospheres of the outer planets or on the surface of their moons. Second, we would like to know what types of meteoroids can survive the entry flight and deliver the building blocks of life to the Earth's surface.

The study of the entry flights of meteoroids leads to the third emerging problem: protection of the Earth from the bombardment of meteoroids. How large a meteoroid should we be afraid of? We do not even know how large was the meteoroid that fell in the Tunguska region of Siberia in 1908. Work must be done to deteermine the characteristics of the meteoroids.

For the fourth emerging problem, one must cite the entry flight into the outer planets. As was mentioned above, there is an amply good reason to explore outer planets and their moons. In order to increase the payload into those celestial bodies, aero-capturing in the outer planets becomes necessary. In order to know the forces and heat transfer rates to these aero-capturing vehicles, thermo-chemistry of the gas mixture consisting of hydrogen, helium, and methane must be studied. This should be considered a new class of entry physics problems.

\section{Future Tasks for Aerothermodynamicists}

From the above, one can see that there are three new directions that aerothermodynamicists must follow. First, try to remove approximately one degree uncertainty in trim angle of attack by improving the non-equilibrium model; second, study the thermochemical phenomena in $\mathrm{H}_{2}+\mathrm{He}+\mathrm{CH}_{4}$ mixture; and third, study the entry flights of meteors.

By removing the remaining one degree uncertainty in trim angle of attack, we may eventually be able to design an efficient hypersonic vehicle. Such a vehicle may one day fly over the Pacific Ocean carrying passengers.

One small point regarding the second task, study of the thermochemistry of a $\mathrm{H}_{2}+\mathrm{He}+\mathrm{CH}_{4}$ mixture, concerns the question as to how one could produce the needed $25 \mathrm{~km} / \mathrm{s}$ in a shock tube. Calculations show that the driver gas is allowed to be heated only up to about $11,000 \mathrm{~K}$. At higher 
temperatures, the radiative heating of the driven gas by the driver gas will occur. In order to produce $25 \mathrm{~km} / \mathrm{s}$ shock speed with a driver consisting of hydrogen heated to $11,000 \mathrm{~K}$, the driver pressure must be in the order of 10,000 bars. There is no metal that can withstand such a high pressure: the driver will have to use an explosive, which will be destroyed each time. Such a disposable driver could be built as shown in Figure 23. Such a shock tube existed in the 1970s.

Theoretical works must accompany these endeavors. Thermochemical and radiation properties must be known for the $\mathrm{H}_{2}+\mathrm{He}+\mathrm{CH}_{4}$ mixture. For the study of ablation of meteoroids, one must know the properties of $\mathrm{SiO}, \mathrm{MgO}$, $\mathrm{FeO}, \mathrm{SO}, \mathrm{Si}, \mathrm{Mg}, \mathrm{Fe}, \mathrm{S}, \mathrm{Si}^{+}, \mathrm{Mg}^{+}, \mathrm{Fe}^{+}$, and $\mathrm{S}^{+}$. For this purpose, aerothermodynamicists should in the future tackle quantum mechanics by themselves. Computer program packages are available today to solve the Schroedinger equation, a task of the theoretical chemists in the past. Ever since these computer codes became available, theoretical chemists are reluctant to carry out this task for aerospace engineers.

One can refer to Ishara here again: the goddess Ishara points the direction of hypersonic aerothermodynamics clearly into the higher, faster, and farther realm. It is the direction pointed by the astronaut Ishara in the Star Trek episode that is imagined to take place in the year 2367 (Figure 24).

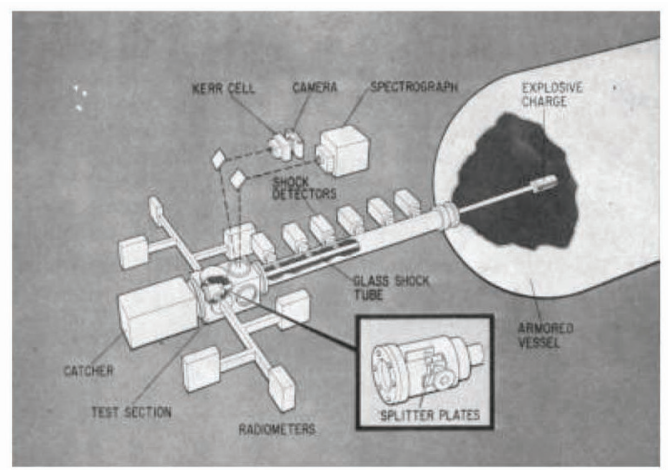

Fig. 23. Explosively-driven shock tube

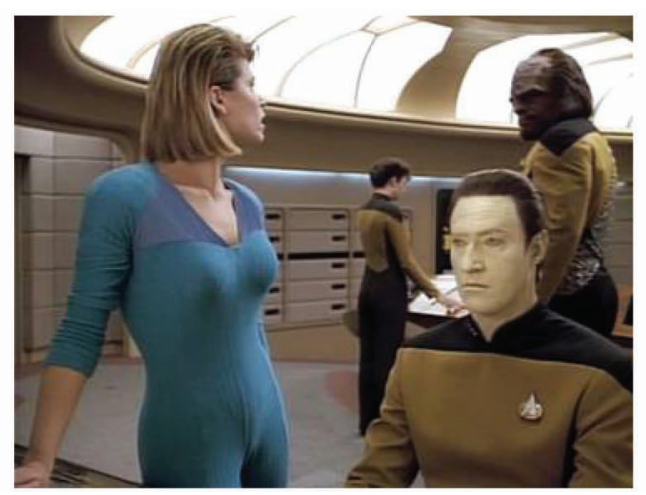

Fig. 24. Astronaut Ishara in a Star Trek episode, 2367

\section{References}

[1] Park, C., Nonequilibrium Hypersonic Aerothermodynamics, John Wiley and Sons, New York, NY, 1990, pp.306312 .

[2] Park, C., Nonequilibrium Hypersonic Aerothermodynamics, John Wiley and Sons, New York, NY, 1990, 312-316.

[3] Sharma, S. P. and Gillespie, W., "Nonequilibrium and Equilibrium Shock Front Radiation Measurements," Journal of Thermophysics and Heat Transfer, Vol. 5, No. 3, 1991, pp. 257-265.

[4] Park, C., Nonequilibrium Hypersonic Aerothermodynamics, John Wiley and Sons, New York, NY, 1990, p.281.

[5] Kim, J. G., and Boyd, I., "Master Equation Analysis of Thermochemical Nonequilibrium of Nitrogen," $43^{\text {rd }}$ AIAA Thermophysics Conference, New Orleans AIAA Paper 20123305, 2012.

[6] Park, C., Nonequilibrium Hypersonic Aerothermodynamics, John Wiley and Sons, New York, NY, 1990, pp.8992.

[7] Holden, M. S., Wadhams, T. P., MacLean, M., Dufrene, A., Mundy, E., and Marineau, E., "A Review of Basic Research and Development Programs Conducted in the LENS Facilities in Hypervelocity Flows," $50^{\text {th }}$ AIAA Aerospace Science Meeting including the New Horizons Forum and Aerospace Exposition, Nashville, AIAA Paper 2012-169, 2012.

[8] Furudate, M., Nonaka, S., and Sawada, K., "Behavior of Two-Temperature Model in Intermediate Hypersonic Regime," Journal of Thermophysics and Heat Transfer, Vol. 13, No. 4, 1999, pp. 424-430.

[9] Fujita, K., Sato, S., Abe, T., and Ebinuma, Y., "Experimental Investigation of Air Radiation From Behind a Strong Shock Wave," Journal of Thermophysics and Heat Transfer, Vol. 16, No. 1, 2002, pp. 77-82.

[10] Dankert, C., "Rarefied Flow Investigation in GasSurface Interaction and Supersonic Plumes," Colloquium on Nonequilibrium Phenomena of Low-Density Jets in Space, Kyoto University, 1998, pp.51-60.

[11] Park, C., "Rotational Relaxation of $\mathrm{N}_{2}$ Behind a Strong Shock Wave," Journal of Thermophysics and Heat Transfer, Vol. 18, No. 4, 2004, pp.527-533.

[12] Matsuyama, S., Ohnishi, N., Sasoh, A., and Sawada, K., "Numerical Simulation of Galileo Probe Entry Flowfield With Radiation and Ablation," Journal of Thermophysics and Heat Transfer, Vol. 19, No. 1, 2005, pp.28-35.

[13] Park, C., "Stagnation-Region Heating Environment of the Galileo Probe," Journal of Thermophysics and Heat Transfer, Vol. 23, No. 3, 2009, pp.417-424. 
[14] Park, C., "Nonequilibrium Chemistry and Radia-tion for Neptune Entry," Journal of Spacecraft and Rockets, Vol. 48, No. 6, 2011, pp.897-903.

[15] Leibowitz, L. P., "Measurement of the Structure of an Ionizing Shock Wave in a Hydrogen-Helium Mixture," The Physics of Fluids, Vol. 16, No. 1, 1973, pp.59-68.

[16] Livingston, F. R., and Poon, T. Y., "Relaxation Distance and Equilibrium Electron Density Measurements in Hydrogen-Helium Plasmas," AIAA Journal, Vol. 14, No. 9, 1976, pp.1335-1337.

[17] Bogdanoff, D. W., and Park, C., "Radiative Interaction Between Driver and Driven Gases in an Arc-Driven Shock Tube," Shock Waves, Vol. 12, No. 1, 2002, pp.205-214.
[18] Omura, M., and Presley, L. L., "Electron Density Measurements Ahead of Shock Waves in Air," AIAA Journal, Vol. 7, No. 12, 1969, pp.2363-2365.'

[19] Park, C., Nonequilibrium Hypersonic Aer-othermodynamics, John Wiley and Sons, New York, NY, 1990, pp.303306.

[20] Wada, Y., Watanabe, Y., Akimoto, T., and Yasui, H. “Data Analysis of Electrostatic Probe," Proceedings of HOPE/ OREX Workshop (in Japanese), Tokyo, paper F-2, 1994.

[21] Park, C., "Injection-Induced Turbulence in Stagnation-Point Boundary Layers," AIAA Journal, Vol. 22, No. 2, 1984, pp.219-225. 